\title{
A BPE Model for the Burgers Equation
}

\author{
By \\ Shigeyoshi OgawA* and Arturo Kohatsu-HigA**
}

\begin{abstract}
We study the BPE (Brownian particle equation) model of the Burgers equation presented in the preceding article [6]. More precisely, we are interested in establishing the existence and uniqueness properties of solutions using probabilistic techniques.
\end{abstract}

\section{$\S 1$. BPE Model for the Burgers Equation}

In this article, we are concerned with the stochastic representation for the Burgers equation. For this purpose we presented in the preceding article [6] the two BPE models, one of which is as follows;

$$
\begin{aligned}
\partial_{t} u(t, x)+\epsilon \dot{W} \partial_{x} u(t, x) & =\frac{1}{\epsilon} f(\bar{u}(t, x)) \dot{W}, \quad f(x)=x^{2} \\
u(0, x) & =u_{0}(x) .
\end{aligned}
$$

Here $t \in[0, T], x \in \mathbb{R}, \bar{u}(t, x)=E(u(t, x))$ and $\dot{W}$ denotes the white noise process. We already know by [6] (see Theorem 2.1) that for $f$ regular (smooth with bounded derivatives) this problem has one and only one solution. This requirement is needed so that one can use classical results in the theory of PDEs.

Here, we would like to establish the result for $f(x)=x^{2}$ via a probabilistic method so as to make the discussion self-contained and this is the principal objective of the present note. More precisely, we are to show that the solution can

Communicated by Y. Takahashi. Received June 14, 2002. Revised November 11, 2002, March 26, 2003.

2000 Mathematics Subject Classification(s): 60H15, 60H30

* Ritsumeikan University, Department of Mathematics.

e-mail: ogawa-s@se.ritsumei.ac.jp

** Universitat Pompeu Fabra, Department of Economics.

e-mail: arturo.kohatsu@econ.upf.es. 
be constructed through a system of stochastic integral equations that can be obtained by applying the method of stochastic characteristics to the Cauchy problem (1). We will in general present a heuristic solution for the following equation

$$
\begin{aligned}
\partial_{t} u(t, x)+(a+\sigma \dot{W}) \partial_{x} u(t, x) & =\nu f(\bar{u}(t, x)) \dot{W}, f(x)=x^{2} \\
u(0, x) & =u_{0}(x) .
\end{aligned}
$$

For two smooth coefficients $a$ and $\sigma$. In particular the above example will follow from using $a=0, \sigma=\varepsilon$ and $\nu=1 / \varepsilon$.

A different stochastic representation has been obtained by Bossy and Talay $[1],[2]$.

\section{§2. Preliminaries - Noncausal Stochastic Calculus}

Since the stochastic integral of noncausal type, introduced by S.Ogawa in 1979 [11], plays an essential role in the BPE theory, we will briefly introduce it following [7] so that the discussion is self-contained. For more details of the noncausal calculus we refer the reader to [7], [9].

\section{§2.1. Causal functions and B-differentiability}

In Itô's theory, the stochastic integral, say with respect to the Brownian motion $\left\{W_{t}(\omega) ; t \in[0, T]\right\}$, denoted by $\int f(t, \omega) d^{+} W_{t}$, is defined only for integrands $f(t, \omega)$ that are causal (or non anticipative) with respect to the history of the Brownian motion. Namely, $f(t, \omega)$ is supposed to be adapted (sometimes also called "causal") to the filtration $\left\{\mathcal{F}_{t}, t \geq 0\right\}$ where the $\mathcal{F}_{t}=\sigma\left\{W_{s} ; 0 \leq\right.$ $s \leq t\}$. In many situations one meets problems of noncausal character, therefore there is a need for another theory of stochastic calculus which is free from the restriction of causality. The noncausal calculus is one of such theories.

In what follows, we will fix the probability space $(\Omega, \mathcal{F}, P)$ on which is defined the $\mathbb{R}$-valued Brownian motion. We denote by $\mathbf{H}$ the totality of all random functions $f(t, \omega)$, measurable in $(t, \omega)$ with respect to the field $\mathcal{B}\left(\mathbb{R}_{+}\right) \otimes$ $\mathcal{F}$, such that $P\left\{\int_{0}^{T}|f(t, \omega)|^{2} d t<\infty\right\}=1$, and by $\mathbf{M}_{p}^{+}$is the subset of $\mathbf{H}$ containing all $L^{p}$-causal random functions, that is, the set of functions $f(t, \omega)$ such that they are;

(M.1) measurable in $(t, \omega)$ with respect to the field $\mathcal{B}\left(\mathbb{R}_{+}\right) \otimes \mathcal{F}$, and especially

(M.2) adapted to the family of $\sigma$-fields $\left\{\mathcal{F}_{t}\right\}$, where $\mathcal{F}_{t}=\sigma\left\{W_{s}\right.$; $0 \leq s \leq t\}$,

(M.3) and has finite moments of order $p, E\left(\int_{0}^{T}|f(t, \omega)|^{p} d t\right)<\infty$. 
An $\mathbf{H}$-class random function $g(t, \omega)$ is said to be differentiable in $L^{p}(\Omega)$ with respect to the Brownian motion $W_{t}$ (or $B_{p}^{+}$-differentiable) provided that $\sup _{t} E|g(t)|^{p}<\infty$ and there exists an $\mathbf{M}_{p}^{+}$-class random function say $\hat{g}(t, \omega)$ such that, for any small enough $h>0$,

$$
\sup _{t, s,|t-s|<h} E\left|g(t, \omega)-g(s, \omega)-\int_{s}^{t} \hat{g}(r, \omega) d^{+} W_{r}\right|^{p}=o\left(h^{p / 2}\right)
$$

where the integral $\int \cdot d^{+} W$ stands for Itô's stochastic integral. The function $\hat{g}(r, \omega)$ is called the $B_{p}^{+}$-derivative ${ }^{1}$ of $g$ and denoted by $\frac{\partial^{+} g}{\partial W(r)}$. It is not difficult to see that if the function $g(t, \omega)$ is $B_{p}^{+}$-differentiable then its $B_{p}^{+}$-derivative is uniquely determined as soon as its derivative is in $L^{2}([0, T] \times \Omega)$ (see [12]) and that it satisfies a chain rule when $g$ is adapted. That is, let $f \in C^{2}$ with bounded second derivative and $g$ be $B_{p}^{+}$differentiable for $p \geq 4$, then the $B_{p / 2}^{+}$-derivative of $f(g)$ exists and we have

$$
\frac{\partial^{+} f(g)}{\partial W(r)}=f^{\prime}(g(r)) \frac{\partial^{+} g}{\partial W(r)} .
$$

The proof of this assertion is not difficult to obtain if one uses Ito's formula appropriately. The $B_{p}^{+}$-differentiability of the random function with respect to the multi-dimensional Brownian motion is defined in a similar way. Also one can define similarly the processes adapted to the filtration of the backward Wiener process, which we will denote by $\mathbf{M}_{p}^{-}$. The backward stochastic integral will be denoted by $\int \cdot d^{-} W_{s}$. Then one defines the $B_{p}^{-}$-derivative with respect to backward integration and in such a case one denotes it by $\frac{\partial^{-} g}{\partial W(r)}$.

Note. The notion of $B$-derivatives and the notations $\frac{\partial^{+}}{\partial W_{t}}, \frac{\partial^{-}}{\partial W_{t}}$ were first introduced by S. Ogawa in [13], and later in [6], [7]. But the definition of the $\frac{\partial^{-}}{\partial W_{t}}$ given in those papers is slightly different from the one given here.

\section{§2.2. Noncausal stochastic integral}

Given a random function $f(t, \omega) \in \mathbf{H}$ and an arbitrary complete orthonormal system $\left\{\phi_{n}\right\}$ in $L^{2}([0, T])$, we consider the formal random series

$$
\sum_{n}^{\infty} \int_{0}^{T} f(t, \omega) \phi_{n}(t) d t \times \int_{0}^{T} \phi_{n}(t) d W_{t} .
$$

The stochastic integral of noncausal type was introduced by S. Ogawa in 1979 [11], in the following form:

\footnotetext{
${ }^{1}$ The notion of the $B_{p}^{+}$-derivative is different from the "stochastic derivative" which was introduced by A. Skorokhod
} 
Definition 1. A random function $f(t, \omega) \in \mathbf{H}$ is said to be integrable with respect to the basis $\left\{\phi_{n}\right\}$ (or $\phi$-integrable) when the random series above converges in probability and the sum, denoted by $\int_{0}^{T} f(t, \omega) d_{\phi} W_{t}$, is called the stochastic integral of noncausal type with respect to the basis $\left\{\phi_{n}\right\}$.

Obviously when $f \in \mathbf{M}_{p}^{+}$for some $p>0$, then the above integral and the stochastic Ito integral coincide.

\section{$\S 2.3$. Relation between symmetric and noncausal integrals}

We call a random function, $f(t, \omega)$, a semimartingale, when it admits the decomposition $f(t, \omega)=a(t, \omega)+\int_{0}^{t} \hat{f}(s) d^{+} W_{s}$ where $\hat{f} \in L^{p}([0, T] \times \Omega)$ for some $p \geq 2$ and $a(\cdot)$ is such that almost every sample path is of bounded variation in $t$ over $[0, T]$. Notice that if $\sup _{t \in[0, T]} E|a(t)|^{p}<\infty$ and $\sup _{t, s|t-s|<h} E|a(t)-a(s)|^{p}=$ $o\left(h^{p / 2}\right)$ then $f$ is $B_{p}^{+}$-differentiable. Note that $a$ does not need to be adapted to the filtration in order to have that $f$ is $B_{p}^{+}$-differentiable and $\frac{\partial^{+} f}{\partial W(t)}=\hat{f}(t)$.

Next we give some basic results about the relation between the symmetric integrals and the noncausal integral. Let $H$ denote the Haar basis.

Theorem 2 ([10]). Every causal $B_{p}^{+}$-differentiable function (for some $p>0)$ is integrable in noncausal sense with respect to the system of Haar functions and the integral coincides with that of the symmetric integral of the same function. That is,

$$
\int_{0}^{T} f(s) d_{H} W_{s}=\int_{0}^{T} f(s) d^{+} W_{s}+\frac{1}{2} \int_{0}^{T} \frac{\partial^{+} f}{\partial W(s)} d s .
$$

A similar relationship is satisfied if $f$ is adapted with respect to the backward filtration except that the stochastic integral becomes the backward stochastic integral which we denote by $\int d^{-} W$ and the Lebesgue integral term has a negative sign in front. The advantage of the above representation is that the integral on the left side of the above equation is always well defined and that we can use it in various situations without having to explain which integral we are using. One can generalize the above integrals to a broader class of basis. We say that a c.o.n.s. $\left\{\phi_{n}\right\}$ is regular provided that it satisfies the next condition:

$$
\sup _{n} \int_{0}^{T}\left|u_{n}(s)\right|^{2} d s<\infty, \quad u_{n}(t)=\sum_{k \leq n} \phi_{k}(t) \int_{0}^{t} \phi_{k}(s) d s .
$$

Theorem 3 ([10]). Every semimartingale (causal or not) is $\phi$-integrable, iff the basis $\left\{\phi_{n}\right\}$ is regular. In this case, the noncausal integral coincides with the symmetric integral. 
From now on we denote the above integral by $\int \cdot d_{\phi} W$ and we will always assume that the basis $\left\{\phi_{n}\right\}$ is regular. Furthermore, we also have the following two equalities

$$
\begin{aligned}
& \int_{0}^{T} f(s) d_{\phi} W_{s}=\int_{0}^{T} f(s) d^{+} W_{s}+\frac{1}{2} \int_{0}^{T} \frac{\partial^{+} f}{\partial W(s)} d s, \text { if } f \in M_{p}^{+} \text {for some } p>0 \\
& \int_{0}^{T} f(s) d_{\phi} W_{s}=\int_{0}^{T} f(s) d^{-} W_{s}-\frac{1}{2} \int_{0}^{T} \frac{\partial^{-} f}{\partial W(s)} d s, \text { if } f \in M_{p}^{-} \text {for some } p>0 .
\end{aligned}
$$

\section{§3. Description of The Stochastic Representation}

In this section we give the main theorem where the method of stochastic characteristics is applied to the case of linear BPEs. This idea could eventually be used to solve a hyperbolic-type stochastic partial differential equation. From now on we denote the essential supremum norm by $|\cdot|_{\infty}$.

Another important ingredient in the description of the stochastic representation is the solution of the stochastic equation

$$
X_{s}^{(t, x)}=x-\int_{s}^{t} a\left(u, X_{u}^{(t, x)}\right) d u-\int_{s}^{t} \sigma\left(u, X_{u}^{(t, x)}\right) d_{\phi} W_{u}(0 \leq s \leq t \leq T, x \in \mathbb{R}) .
$$

The solution $X$ exists for Lipschitz coefficients. If the coefficients are smooth with bounded derivatives then its density function $p_{t, x}(s, y)$ exists, is smooth and has an upper bound of Gaussian type due to the uniform ellipticity of $\sigma$. That is, for $s<t \leq T$ and any $\alpha, \beta \in \mathrm{N}$, there exists a positive constant $M=M(\alpha, \beta)$ such that we have (see [3], page 261 for details)

$$
\begin{aligned}
\left|\frac{\partial^{\alpha+\beta} p_{t, x}}{\partial x^{\alpha} \partial y^{\beta}}(s, y)\right| & \leq \frac{M \exp \left(-\frac{|x-y|^{2}}{M(t-s)}\right)}{\sqrt{2 \pi}(t-s)^{(\alpha+\beta+1) / 2}}, \\
\left|\frac{\partial^{\alpha+\beta} p_{t, y+x}(s, y)}{\partial x^{\alpha} \partial y^{\beta}}\right| & \leq \frac{M \exp \left(-\frac{|x|^{2}}{M(t-s)}\right)}{\sqrt{2 \pi}(t-s)^{(\alpha+1) / 2}} .
\end{aligned}
$$

We assume without loss of generality that the constant $M$ is increasing in $\alpha$ and $\beta$. Furthermore $p$ satisfies the parabolic PDE

$$
\frac{\partial p_{t, x}}{\partial t}(s, y)=\frac{\sigma^{2}(t, x)}{2} \frac{\partial^{2} p_{t, x}}{\partial x^{2}}(s, y)+b(t, x) \frac{\partial p_{t, x}}{\partial x}(s, y)
$$

where $b(t, x)=a(t, x)-\frac{1}{2} \sigma \sigma^{\prime}(t, x)$.

Theorem 4. Suppose that the initial data $u_{0}(x)$ satisfies the condition,

$$
u_{0} \in C^{2} \text { and that }\left|u_{0}\right|_{\infty}+\left|u_{0}^{\prime}\right|_{\infty}+\left|u_{0}^{\prime \prime}\right|_{\infty}<C(0) .
$$


Furthermore suppose that $a(t, \cdot), \sigma(t, \cdot) \in C_{b}^{\infty}(\mathbb{R})$ uniformly in $t \in[0, T]$ and that there exists positive constants $c_{0}$ and $c$ such that for all $(t, x) \in[0, T] \times \mathbb{R}$, $|\sigma(t, x)| \geq c_{0}>0$ and $|\sigma(t, \cdot)|_{\infty}+\left|\sigma^{\prime}(t, \cdot)\right|_{\infty}<c$. Then if $c \nu C(0) M^{3 / 2}(2,2)$ is small enough then the integral equations

$$
u(t, x)=u_{0}\left(X_{0}^{(t, x)}\right)+\nu \int_{0}^{t} \bar{u}\left(s, X_{s}^{(t, x)}\right)^{2} d_{\phi} W_{s}
$$

have only one global solution $u$. The average of $u, \bar{u}(t, x)=E u(t, x)$ solves the following Cauchy problem for the Burgers equation:

$$
\begin{aligned}
\frac{\partial \bar{u}}{\partial t}+\nu \sigma(t, x) \frac{\partial \bar{u}^{2}}{\partial x}(t, x) & =\frac{\sigma(t, x)^{2}}{2} \frac{\partial^{2} \bar{u}}{\partial x^{2}}(t, x)+b(t, x) \frac{\partial \bar{u}}{\partial x}(t, x) \\
\bar{u}(0, x) & =u_{0}(x) .
\end{aligned}
$$

$M$ is a constant that will depend on the smoothness of the coefficients $a$ and $\sigma$ as well as in the constant $c_{0}$. The restriction on the size of $c \nu C(0) M^{3 / 2}(2,2)$ can be explicitly characterized but as it depends on other constants that appear later in the proofs we will state it explicitly in the proof of Lemma 6 .

Note that in the above theorems the integral $\int \cdot d_{\phi} W_{s}$ is the stochastic integral of noncausal type which, applied to the causal (or non anticipating) integrand, coincides with the integrals of symmetric type (i.e. the so called Stratonovich's integral or the $\mathcal{I}_{1 / 2}$ integral introduced by S. Ogawa [13]). Suppose that $f(\bar{u})(t, x)=f(E(u(t, x)))$ is twice differentiable in $x$ with bounded second derivative, then we have the next relation, as $X^{(t, x)} \in \mathbf{M}_{p}^{-}$, for any $p>0$

$$
\begin{aligned}
& \int_{0}^{t} f(\bar{u})\left(s, X_{s}^{(t, x)}\right) d_{\phi} W_{s} \\
& \quad=\int_{0}^{t} f(\bar{u})\left(s, X_{s}^{(t, x)}\right) d^{-} W_{s}-\frac{1}{2} \int_{0}^{t} \frac{\partial^{-} f(\bar{u})\left(\cdot, X^{(t, x)}\right)}{\partial W(s)} d s .
\end{aligned}
$$

We easily see from the equations above that the $B_{p}^{-}$-derivative of the integrand is given by the following (using the chain rule)

$$
\begin{aligned}
\frac{\partial^{-} f(\bar{u})\left(\cdot, X^{(t, x)}\right)}{\partial W_{s}} & =\left(f^{\prime}(\bar{u}) \frac{\partial \bar{u}}{\partial x}\right)\left(s, X_{s}^{(t, x)}\right) \frac{\partial^{-} X^{(t, x)}}{\partial W_{s}} \\
& =\left(f^{\prime}(\bar{u}) \frac{\partial \bar{u}}{\partial x} \sigma\right)\left(s, X_{s}^{(t, x)}\right) .
\end{aligned}
$$


Therefore

$$
\begin{aligned}
& \int_{0}^{t} f(\bar{u})\left(s, X_{s}^{(t, x)}\right) d_{\phi} W_{s} \\
& \quad=\int_{0}^{t} f(\bar{u})\left(s, X_{s}^{(t, x)}\right) d^{-} W_{s}-\frac{1}{2} \int_{0}^{t} f^{\prime}(\bar{u}) \frac{\partial \bar{u}}{\partial x} \sigma\left(s, X_{s}^{(t, x)}\right) d s .
\end{aligned}
$$

\section{§4. Proof of the Main Theorem}

From now we use the following notation for the constants which will be fixed throughout the proof. In particular, we fix $|\sigma|_{\infty}+\left|\sigma^{\prime}\right|_{\infty}<c,\left|u_{0}\right|_{\infty}+$ $\left|u_{0}^{\prime}\right|_{\infty}+\left|u_{0}^{\prime \prime}\right|_{\infty}<C(0)$. We carry out this proof in several steps:

\section{§4.1. Successive approximation}

For the construction of the solution we apply the Picard's method to our integral equations. Let $\left\{u_{k}\right\}$ be a sequence of random functions defined inductively by

$$
u_{k+1}(t, x)=u_{0}\left(X^{(t, x)}(0)\right)+\nu \int_{0}^{t} \bar{u}_{k}^{2}\left(s, X^{(t, x)}(s)\right) d_{\phi} W_{s} \quad(k \geq 0),
$$

where $u_{0}(t, x)=u_{0}(x)$.

The following lemma states some simple differentiability properties of $\bar{u}_{k}(t, x)=$ $E\left(u_{k}(t, x)\right)$ in order to be able to apply the chain rule. Later in Lemmas 7 and 8 these properties will be refined.

Lemma 5. If $u_{0} \in C^{2}(\mathbb{R})$ with $\left|u_{0}\right|_{\infty}+\left|u_{0}^{\prime}\right|_{\infty}+\left|u_{0}^{\prime \prime}\right|_{\infty}<C(0)$, then $\bar{u}_{k} \in C^{1,2}([0, T] \times \mathbb{R}),\left|\bar{u}_{k}\right|_{\infty}+\left|\bar{u}_{k}^{\prime}\right|_{\infty}+\left|\bar{u}_{k}^{\prime \prime}\right|_{\infty}<C(k) \forall k$ for some positive constants $C(k)$.

Proof. The proof is done by induction. Suppose that the result is true for $k$. Then one has by the chain rule that for fixed $(t, x)$

$$
\frac{\partial^{-} \bar{u}_{k}^{2}\left(\cdot, X^{(t, x)}(\cdot)\right)}{\partial W_{s}}=2 \bar{u}_{k} \bar{u}_{k}^{\prime}\left(s, X^{(t, x)}(s)\right) \frac{\partial^{-} X^{(t, x)}(\cdot)}{\partial W_{s}}=2 \sigma \bar{u}_{k} \bar{u}_{k}^{\prime}\left(s, X^{(t, x)}(s)\right)
$$

where $\bar{u}_{k}^{\prime}(s, y)=\frac{\partial \bar{u}_{k}}{\partial y}(s, y)$. Therefore we have the following representation for the stochastic integral term in (6),

$$
\begin{aligned}
& \int_{0}^{t} \bar{u}_{k}^{2}\left(s, X^{(t, x)}(s)\right) d_{\phi} W_{s} \\
& \quad=\int_{0}^{t} \bar{u}_{k}^{2}\left(s, X^{(t, x)}(s)\right) d^{-} W_{s}-\int_{0}^{t} \sigma \bar{u}_{k} \bar{u}_{k}^{\prime}\left(s, X^{(t, x)}(s)\right) d s .
\end{aligned}
$$


Taking this into account, we get from equation (6) the following equation for the average $\bar{u}_{k}(t, x)=E u_{k}(t, x)$;

$$
\begin{aligned}
\bar{u}_{k+1}(t, x) & =E u_{0}\left(X^{(t, x)}(0)\right)-\nu E \int_{0}^{t} \sigma \bar{u}_{k} \bar{u}_{k}^{\prime}\left(s, X^{(t, x)}(s)\right) d s \\
& =E u_{0}\left(X^{(t, x)}(0)\right)-\nu \int_{0}^{t} \int_{\mathbb{R}} \sigma \bar{u}_{k} \bar{u}_{k}^{\prime}(s, y) p_{t, x}(s, y) d y d s \\
& =E u_{0}\left(X^{(t, x)}(0)\right)+\frac{\nu}{2} \int_{0}^{t} \int_{\mathbb{R}} \bar{u}_{k}(s, y)^{2} \frac{\partial\left(\sigma p_{t, x}\right)}{\partial y}(s, y) d y d s .
\end{aligned}
$$

The last equality follows because $\lim _{y \rightarrow \infty} \bar{u}_{k}^{2}(s, y) p_{t, x}(s, y)=0$, for each fixed $k, s<t$ and $x$. Therefore one has that

$$
\left|\bar{u}_{k+1}\right|_{\infty} \leq\left(C(0)+\nu M^{3 / 2} C(k)^{2} c(t+\sqrt{t})\right) .
$$

Now we differentiate with respect to $x$ the second equality in (7) to obtain that

$$
\bar{u}_{k+1}^{\prime}(t, x)=\frac{\partial}{\partial x} E u_{0}\left(X^{(t, x)}(0)\right)-\nu \int_{0}^{t} \int_{\mathbb{R}} \sigma \bar{u}_{k} \bar{u}_{k}^{\prime}(s, y) \frac{\partial p_{t, x}}{\partial x}(s, y) d y d s
$$

Therefore one obtains bounds for $\left|\bar{u}_{k+1}^{\prime}\right|_{\infty}$ similarly. In preparation for the estimates of $\bar{u}_{k+1}^{\prime \prime}$ note that

$$
\frac{\partial p_{t, x}(s, u+x)}{\partial x}=\frac{\partial p_{t, x}}{\partial x}(s, u+x)+\frac{\partial p_{t, x}}{\partial y}(s, u+x) .
$$

For this "diagonal" derivative we will apply the estimate (2). Using a change of variables $(y=u+x)$, the above equality and an integration by parts we have

$$
\begin{aligned}
& \int_{0}^{t} \int_{\mathbb{R}} \sigma \bar{u}_{k} \bar{u}_{k}^{\prime}(s, u+x) \frac{\partial p_{t, x}}{\partial x}(s, u+x) d u d s \\
& =\int_{0}^{t} \int_{\mathbb{R}} \frac{\partial\left(\sigma \bar{u}_{k} \bar{u}_{k}^{\prime}\right)}{\partial y}(s, u+x) p_{t, x}(s, u+x) d u d s \\
& \quad+\int_{0}^{t} \int_{\mathbb{R}} \sigma \bar{u}_{k} \bar{u}_{k}^{\prime}(s, u+x) \frac{\partial p_{t, x}(s, u+x)}{\partial x} d u d s .
\end{aligned}
$$


Differentiating this again we find that

$$
\begin{aligned}
\left|\bar{u}_{k+1}^{\prime \prime}(t, x)\right| \leq & \left|\frac{\partial^{2}}{\partial x^{2}} E u_{0}\left(X^{(t, x)}(0)\right)\right| \\
& +\left|\nu \int_{0}^{t} \int_{\mathbb{R}} \frac{\partial\left(\sigma \bar{u}_{k} \bar{u}_{k}^{\prime}\right)}{\partial y}(s, y) \frac{\partial}{\partial x} p_{t, x}(s, y) d y d s\right| \\
& +\left|\nu \int_{0}^{t} \int_{\mathbb{R}} \frac{\partial\left(\sigma \bar{u}_{k} \bar{u}_{k}^{\prime}\right)}{\partial y}(s, u+x) \frac{\partial p_{t, x}(s, u+x)}{\partial x} d u d s\right| \\
& +\left|\nu \int_{0}^{t} \int_{\mathbb{R}} \sigma \bar{u}_{k} \bar{u}_{k}^{\prime}(s, u+x) \frac{\partial^{2} p_{t, x}(s, u+x)}{\partial x^{2}} d u d s\right|
\end{aligned}
$$

On the other hand, using properties of the derivatives of the flow $X^{(t, x)}(0)$ and the hypotheses on $u_{0}$, one obtains that there is a positive constant $C$ such that $\left|\frac{\partial^{2}}{\partial x^{2}} E u_{0}\left(X^{(t, x)}(0)\right)\right| \leq C$. Finally using inequality (2) we have

$$
\begin{aligned}
\left|\bar{u}_{k+1}^{\prime \prime}(t, x)\right| \leq & C+\nu \int_{0}^{t} \int_{\mathbb{R}} 3 c C(k)^{2} M \frac{\exp \left(-\frac{|x-y|^{2}}{M(t-s)}\right)}{\sqrt{2 \pi}(t-s)} d y d s \\
& +\nu \int_{0}^{t} \int_{\mathbb{R}} 4 c C(k)^{2} M \frac{\exp \left(-\frac{|u|^{2}}{M(t-s)}\right)}{\sqrt{2 \pi}(t-s)^{1 / 2}} d u d s .
\end{aligned}
$$

Therefore

$$
\left|\bar{u}_{k+1}^{\prime \prime}\right|_{\infty} \leq\left(C+\nu M^{3 / 2} C(k)^{2} c(4 t+6 \sqrt{t})\right)
$$

\section{§4.2. Lemmas}

We will show the convergence of the sequence $\left\{\bar{u}_{k}\right\}$. The key is to show the next two lemmas.

Lemma 6. For any two positive constants $C(0)$ and $c$ such that $c \nu M^{3 / 2}$ $C(0)$ is small enough and any $0<t_{0}<1$ the following Volterra equation has a unique bounded solution $y(t)$ up to $t \leq t_{0}$

$$
y(t)=C(0)+\frac{c \nu}{2} M^{3 / 2} \int_{0}^{t} y^{2}(s)\left(1+\frac{1}{\sqrt{t-s}}\right) d s .
$$


Proof. Put $y(t)=\sum_{k=0}^{\infty} \alpha_{k} t^{k / 2}$ and substitute this into the equation (8), then we will have the equality as follows,

$$
\begin{aligned}
\sum_{k=0}^{\infty} \alpha_{k} t^{k / 2}= & C(0)+\frac{c \nu}{2} M^{3 / 2} \int_{0}^{t}\left(\sum_{k=0}^{\infty} \alpha_{k} s^{k / 2}\right)^{2}\left(1+\frac{1}{\sqrt{t-s}}\right) d s \\
= & C(0)+\frac{c \nu}{2} M^{3 / 2} \sum_{k_{1}, k_{2}=0}^{\infty} \alpha_{k_{1}} \alpha_{k_{2}} B\left(\frac{k_{1}+k_{2}}{2}+1, \frac{1}{2}\right) t^{\frac{k_{1}+k_{2}+1}{2}} \\
& +c \nu M^{3 / 2} \sum_{k_{1}, k_{2}=0}^{\infty} \frac{\alpha_{k_{1}} \alpha_{k_{2}}}{k_{1}+k_{2}+2} t^{\frac{k_{1}+k_{2}}{2}+1} .
\end{aligned}
$$

Comparing the coefficients on both sides of the equation above, we can find the coefficients $\alpha_{k}$ as $\alpha_{0}=C(0)$,

$$
\alpha_{k}=\frac{c \nu}{2} M^{3 / 2} B\left(\frac{k+1}{2}, \frac{1}{2}\right) \sum_{k_{1}+k_{2}+1=k} \alpha_{k_{1}} \alpha_{k_{2}}+\frac{c \nu M^{3 / 2}}{k} \sum_{k_{1}+k_{2}+2=k} \alpha_{k_{1}} \alpha_{k_{2}} .
$$

On the other hand, we can see (using Stirling's approximation for the Gamma function, see the appendix) that

$$
\lim _{k \rightarrow \infty} \sqrt{k+1} \cdot B\left(\frac{k+1}{2}, \frac{1}{2}\right)=\sqrt{2 \pi}
$$

and that

$$
\begin{aligned}
& \lim _{k \rightarrow \infty} \sum_{k_{1}+k_{2}+1=k} \frac{1}{\sqrt{k_{1}+1}} \frac{1}{\sqrt{k_{2}+1}} \\
& =\lim _{k \rightarrow \infty} \frac{1}{k} \sum_{k_{1}+k_{2}+1=k} \frac{1}{\sqrt{\frac{k_{1}+1}{k}} \sqrt{\frac{k_{2}+1}{k}}}=\int_{0}^{1} \frac{d x}{\sqrt{x(1-x)}}=\pi .
\end{aligned}
$$

Hence we see that there exists positive constants $\gamma_{1}, \gamma_{2}$ such that for any $k$, we have the estimates,

$$
B\left(\frac{k+1}{2}, \frac{1}{2}\right) \leq \gamma_{1} \frac{1}{\sqrt{k+1}}, \sum_{k_{1}+k_{2}+1=k} \frac{1}{\sqrt{k_{1}+1}} \frac{1}{\sqrt{k_{2}+1}} \leq \gamma_{2} \pi
$$

Given these, we claim that $\alpha_{k} \leq \frac{C(0)}{\sqrt{k+1}}$ for any $k \geq 0$. In fact, for $k=0$ and $k=1$ the result is trivial if $\sqrt{2} c \nu M^{3 / 2} C(0)<1\left(\alpha_{1}=c \nu M^{3 / 2} C(0)^{2}\right)$.

Now suppose the result is valid up to $k-1$ and that $c \nu M^{3 / 2} C(0) \gamma_{2} \pi$ $\left(\frac{\gamma_{1}}{2}+\sqrt{\frac{3}{4}}\right)<1$ then we get from (9) the following inequality that proves the 
assertion;

$$
\begin{aligned}
\alpha_{k} \leq & \frac{c \nu}{2} M^{3 / 2} C(0)^{2} B\left(\frac{k+1}{2}, \frac{1}{2}\right) \sum_{k_{1}+k_{2}+1=k} \frac{1}{\sqrt{\left(k_{1}+1\right)\left(k_{2}+1\right)}} \\
& +\frac{c \nu M^{3 / 2} C(0)^{2}}{k} \sum_{k_{1}+k_{2}+2=k} \frac{1}{\sqrt{\left(k_{1}+1\right)\left(k_{2}+1\right)}} \\
\leq & \left(\frac{c \nu M^{3 / 2} C(0)^{2} \gamma_{1}}{2 \sqrt{k+1}}+\frac{c \nu M^{3 / 2} C(0)^{2}}{k}\right) \gamma_{2} \pi \leq \frac{C(0)}{\sqrt{k+1}} .
\end{aligned}
$$

Note that the restriction on the constant $c \nu M^{3 / 2} C(0)$ is explicit and fixed by the constants $\gamma_{1}$ and $\gamma_{2}$.

The series

$$
\sum_{k} \alpha_{k} t^{k / 2} \leq C(0)\left(1+\sum_{k \geq 1} \frac{t^{k / 2}}{\sqrt{k+1}}\right)
$$

converges absolutely over the interval $\left[0, t_{0}\right]$ with $t_{0}<1$ and this completes the proof of existence. For uniqueness one follows the usual proof of taking the difference between the two solutions and using the boundedness together with Gronwall's inequality.

Lemma 7. For small enough $t\left(\leq t_{0}\right)$ it holds that $\sup _{t \in\left[0, t_{0}\right], x, k}\left|\bar{u}_{k}(t, x)\right|$ $\leq C_{1}$ where $C_{1}=C(0)\left(1+A_{\beta}\left(t_{0}\right)\right)$ where $A_{\beta}\left(t_{0}\right)=\sum_{k \geq 1} \frac{t_{0}^{k / 2}}{\sqrt{k+1}}$.

Proof. From the equation (7) we get,

$$
\left|\bar{u}_{k+1}(t, x)\right| \leq C(0)+\frac{\nu}{2} \int_{0}^{t} \int_{\mathbb{R}} \bar{u}_{k}^{2}(s, y)\left|\frac{\partial}{\partial y}\left(\sigma p_{t, x}\right)(s, y)\right| d y d s .
$$

Since

$$
\left|\left(\sigma^{\prime} p_{t, x}+\sigma \frac{\partial}{\partial y} p_{t, x}\right)(s, y)\right| \leq \frac{c}{\sqrt{2 \pi(t-s)}}\left(M+\frac{M}{\sqrt{t-s}}\right) \exp \left(-\frac{|x-y|^{2}}{M(t-s)}\right),
$$

then one has that

$$
\int_{\mathbb{R}}\left|\frac{\partial}{\partial y}\left(\sigma p_{t, x}\right)(s, y)\right| d y \leq c M^{3 / 2}\left(1+\frac{1}{\sqrt{t-s}}\right) .
$$

This, in turn, implies the following inequality,

$$
\sup _{x}\left|\bar{u}_{k+1}(t, x)\right| \leq C(0)+\frac{c \nu M^{3 / 2}}{2} \int_{0}^{t}\left\{\sup _{y} \bar{u}_{k}^{2}(s, y)\right\}\left(1+\frac{1}{\sqrt{t-s}}\right) d s .
$$


The next step consists of proving that for any $k \in N$, we have that

$$
\sup _{x}\left|\bar{u}_{k}(t, x)\right| \leq y(t) \quad\left(\forall t \leq t_{0}\right) .
$$

By applying induction we have first that $\sup _{x}\left|\bar{u}_{0}(t, x)\right| \leq C(0) \leq y(t)$. Now suppose the inequality is satisfied for $k$. Then using the above inequality and the previous Lemma 6 we have that the inequality is satisfied for $k+1$.

Following a similar discussion to that given in the proof of the Lemma 6 , we can establish the following result;

Lemma 8. For small enough $t\left(\leq t_{0}\right)$, there exists a positive constant $C_{2}$ such that $\sup _{t \in\left[0, t_{0}\right], x, k}\left|\bar{u}_{k}^{\prime}(t, x)\right|<C_{2}$.

Proof. First, one differentiates (7) to obtain

$$
\begin{aligned}
\bar{u}_{k+1}^{\prime}(t, x)= & E\left(u_{0}^{\prime}\left(X^{(t, x)}(0)\right) \frac{\partial X^{(t, x)}(0)}{\partial x}\right) \\
& -\nu \int_{0}^{t} \int_{\mathbb{R}} \sigma \bar{u}_{k} \bar{u}_{k}^{\prime}(s, y) \frac{\partial}{\partial x}\left(p_{t, x}\right)(s, y) d y d s .
\end{aligned}
$$

As before the proof follows by induction. First we have that $\sup _{x, t \in\left[0, t_{0}\right]}\left|\bar{u}_{0}^{\prime}(t, x)\right|$ $\leq C(0)$. Then we have using the previous Lemma that there exists a positive constant $A\left(C(0), t_{0}\right)$ such that

$$
\sup _{x}\left|\bar{u}_{k+1}^{\prime}(t, x)\right| \leq A\left(C(0), t_{0}\right)+c C_{1} \nu M^{3 / 2} \int_{0}^{t} \sup _{x}\left|\bar{u}_{k}^{\prime}(s, x)\right|\left(1+\frac{1}{\sqrt{t-s}}\right) d s .
$$

Using the Gronwall lemma in the Appendix, one finally obtains that

$$
\sup _{x}\left|\bar{u}_{k+1}^{\prime}(t, x)\right| \leq A\left(C(0), t_{0}\right) \exp \left((t+2 \sqrt{t}) c C_{1} \nu M^{3 / 2}\right) .
$$

Similarly, one obtains the same conclusion for the second derivative. That is, $\sup _{t \in\left[0, t_{0}\right], x, k}\left|\bar{u}_{k}^{\prime \prime}(t, x)\right|$ is bounded. Without loss of generality we assume that $C_{2}>C_{1}$.

\section{§4.3. Convergence of the sequence $\left\{\bar{u}_{k}\right\}$}

Proposition 9. The sequence $\left\{\bar{u}_{k}(t, x)\right\}$ converges to $\bar{u}(t, x)$ uniformly in $(t, x) \in\left[0, t_{0} \wedge 1\right] \times \mathbb{R}$ as $k \rightarrow \infty$. Here $\bar{u}(t, x)$ is the unique solution to the integral equation

$$
\bar{u}(t, x)=E u_{0}\left(X^{(t, x)}(0)\right)+\frac{\nu}{2} \int_{0}^{t} \int_{\mathbb{R}} \bar{u}^{2}(s, y) \frac{\partial}{\partial y}\left(\sigma p_{t, x}\right)(s, y) d y d s .
$$


Proof. From the equation (7) and Lemma 7, we get for $t \leq t_{0} \wedge 1$ the following,

$$
\begin{aligned}
\left|\bar{u}_{k}(t, x)-\bar{u}_{k-1}(t, x)\right| & =\frac{\nu}{2}\left|\int_{0}^{t} \int_{\mathbb{R}}\left[\left\{\left(\bar{u}_{k-1}^{2}\right)^{\prime}-\left(\bar{u}_{k-2}^{2}\right)^{\prime}\right\} \sigma p_{t, x}\right](s, y) d y d s\right| \\
& =\frac{\nu}{2}\left|\int_{0}^{t} \int_{\mathbb{R}}\left[\left\{\bar{u}_{k-1}^{2}-\bar{u}_{k-2}^{2}\right\} \frac{\partial}{\partial y}\left(\sigma p_{t, x}\right)\right](s, y) d y d s\right| \\
& \leq C_{1} \nu \int_{0}^{t} \int_{\mathbb{R}}\left|\bar{u}_{k-1}-\bar{u}_{k-2}\right|(s, y)\left|\frac{\partial}{\partial y}\left(\sigma p_{t, x}\right)(s, y)\right| d y d s .
\end{aligned}
$$

Put $B_{k}(t)=\sup _{x}\left|\bar{u}_{k}(t, x)-\bar{u}_{k-1}(t, x)\right|$, then from the inequality above we obtain,

$$
\begin{aligned}
B_{k}(t) & \leq C_{1} \nu \int_{0}^{t} B_{k-1}(s) \int_{\mathbb{R}}\left|\frac{\partial}{\partial y}\left(\sigma p_{t, x}\right)(s, y)\right| d y d s \\
& \leq C_{1} c \nu M^{3 / 2} \int_{0}^{t} B_{k-1}(s)\left(1+\frac{1}{\sqrt{t-s}}\right) d s .
\end{aligned}
$$

Hence by induction we get the inequality as follows for some positive constant $C_{3}$,

$$
B_{k}(t) \leq C_{3}^{k}\left\{\prod_{j=2}^{k} B\left(\frac{j+1}{2}, \frac{1}{2}\right)\right\} t^{\frac{k}{2}} .
$$

In fact, for $k=1$, one has that

$$
\begin{aligned}
B_{1}(t) & \leq\left|E\left(u_{0}\left(X_{0}^{(t, x)}\right)-u_{0}(x)\right)\right|+\int_{0}^{t} \sup _{x}\left|\bar{u}_{0}(s, x)\right|^{2}\left(1+\frac{1}{\sqrt{t-s}}\right) d s \\
& \leq C_{4} \sqrt{t}
\end{aligned}
$$

for some positive constant $C_{4}$. Now we can rewrite inequality (11) as

$$
B_{k}(t) \leq 2 C_{1} c \nu M^{3 / 2} \int_{0}^{t} \frac{B_{k-1}(s)}{\sqrt{t-s}} d s .
$$

Here we take $C_{4} \vee\left(2 C_{1} c \nu M^{3 / 2}\right) \leq C_{3}$. Supposing that (12) is satisfied up to $k-1$, we have

$$
\begin{aligned}
B_{k}(t) & \leq 2 C_{1} c \nu M^{3 / 2} \int_{0}^{t} \frac{C_{3}^{k-1}\left\{\prod_{j=2}^{k-1} B\left(\frac{j+1}{2}, \frac{1}{2}\right)\right\} s^{\frac{k-1}{2}}}{\sqrt{t-s}} d s \\
& \leq C_{3}^{k}\left\{\prod_{j=2}^{k-1} B\left(\frac{j+1}{2}, \frac{1}{2}\right)\right\} t^{\frac{k}{2}} \int_{0}^{1} \frac{s^{\frac{k-1}{2}}}{\sqrt{1-s}} d s .
\end{aligned}
$$


Since

$$
\lim _{k \rightarrow \infty} \frac{C_{3}^{k}\left\{\prod_{j=2}^{k} B\left(\frac{j+1}{2}, \frac{1}{2}\right)\right\}}{C_{3}^{k+1}\left\{\prod_{j=2}^{k+1} B\left(\frac{j+1}{2}, \frac{1}{2}\right)\right\}}=\lim _{k \rightarrow \infty} \frac{1}{C_{3} B\left(\frac{k+2}{2}, \frac{1}{2}\right)}=\infty,
$$

we see that the series $\sum_{k} B_{k}(t)$ converges uniformly for $t<t_{0} \wedge 1$. This finishes the proof.

Following the similar discussion and taking the Lemma 8 into account, we also establish the next result.

Proposition 10. The sequence $\left\{\bar{u}_{k}^{\prime}(t, x)\right\}$ converges uniformly on any finite slab $\left[0, t_{0}\right] \times \mathbb{R}$ to $\bar{u}^{\prime}$ as $k$ tends to $\infty$.

Proof. As before,

$$
\begin{aligned}
& \left|\bar{u}_{k}^{\prime}(t, x)-\bar{u}_{k-1}^{\prime}(t, x)\right| \\
& =\nu\left|\int_{0}^{t} \int_{\mathbb{R}}\left[\sigma\left\{\bar{u}_{k-1} \bar{u}_{k-1}^{\prime}-\bar{u}_{k-2} \bar{u}_{k-2}^{\prime}\right\} \frac{\partial}{\partial x}\left(p_{t, x}\right)\right](s, y) d y d s\right| \\
& \leq C_{2} c \nu \int_{0}^{t} \int_{\mathbb{R}}\left|\bar{u}_{k-1}-\bar{u}_{k-2}\right|(s, y)\left|\frac{\partial}{\partial x}\left(p_{t, x}\right)(s, y)\right| d y d s \\
& \quad+C_{1} c \nu \int_{0}^{t} \int_{\mathbb{R}}\left|\bar{u}_{k-1}^{\prime}-\bar{u}_{k-2}^{\prime}\right|(s, y)\left|\frac{\partial}{\partial x}\left(p_{t, x}\right)(s, y)\right| d y d s .
\end{aligned}
$$

Define $B_{k}^{\prime}(t)=\sup _{x}\left|\bar{u}_{k}^{\prime}(t, x)-\bar{u}_{k-1}^{\prime}(t, x)\right|$. Then we have

$$
B_{k}^{\prime}(t) \leq 2 C_{2} c \nu M^{3 / 2}\left(\int_{0}^{t} \frac{B_{k-1}(s)}{\sqrt{t-s}} d s+\int_{0}^{t} \frac{B_{k-1}^{\prime}(s)}{\sqrt{t-s}} d s\right) .
$$

As before one also proves in this case that $\sum_{k} B_{k}^{\prime}(t)$ converges uniformly for $t<t_{0} \wedge 1$. Denote the limit of $\bar{u}_{k}^{\prime}$ by $\bar{v}$. Then it satisfies the equation

$$
\bar{v}(t, x)=E u_{0}\left(X^{(t, x)}(0) \frac{\partial X^{(t, x)}(0)}{\partial x}\right)+\nu \int_{0}^{t} \int_{\mathbb{R}}(\bar{u} \bar{v})(s, y) \frac{\partial}{\partial x}\left(\sigma p_{t, x}\right)(s, y) d y d s .
$$

The convergence being uniform also gives that $\bar{u}$ is differentiable in $x$. The above equation has a unique solution which is also satisfied by $\bar{u}^{\prime}$ if one differentiates (10). This finishes the proof.

Based on these Propositions, we let $k$ tend to infinity on both sides of the first equation in (7) to find that the $\lim _{k} \bar{u}_{k}=\bar{u}$ is the solution of the following,

$$
\bar{u}(t, x)=E u_{0}\left(X^{(t, x)}(0)\right)-\nu \int_{0}^{t} \int_{\mathbb{R}} \bar{u} \bar{u}^{\prime}(s, y) \sigma p_{t, x}(s, y) d y d s .
$$


Proposition 11. The solution, bounded in $x$, of the equation (13) is unique.

Proof. Let $\bar{u}, \bar{v}$ be the solutions that are bounded in $x$. Then we have,

$$
|\bar{u}(t, x)-\bar{v}(t, x)| \leq \frac{\nu}{2} \int_{0}^{t} \int_{\mathbb{R}}\left|\bar{u}^{2}(s, y)-\bar{v}^{2}(s, y)\right|\left|\frac{\partial}{\partial y}\left(\sigma p_{t, x}\right)(s, y)\right| d y d s .
$$

Hence,

$$
\sup _{x}|\bar{u}(t, x)-\bar{v}(t, x)| \leq C_{1} c \nu M^{3 / 2} \int_{0}^{t} \sup _{y}|\bar{u}(s, y)-\bar{v}(s, y)| \frac{1}{\sqrt{t-s}} d s .
$$

By applying the Gronwall's lemma (see the Appendix) to this inequality we get the conclusion.

Similarly as in the previous arguments one can also prove the convergence and existence of the second derivative of $\bar{u}(t, x)$. In fact, one has that

$$
\begin{aligned}
& \left|\bar{u}_{k}^{\prime \prime}(t, x)-\bar{u}_{k-1}^{\prime \prime}(t, x)\right| \\
& \leq\left|\nu \int_{0}^{t} \int_{\mathbb{R}} \frac{\partial}{\partial y}\left(\sigma\left(\bar{u}_{k-1} \bar{u}_{k-1}^{\prime}-\bar{u}_{k-2} \bar{u}_{k-2}^{\prime}\right)\right)(s, y)\left(\frac{\partial}{\partial y}+\frac{\partial}{\partial x}\right) p_{(t, x)}(s, y) d y d s\right| \\
& \quad+\nu \int_{0}^{t} \int_{\mathbb{R}}\left|\sigma\left(\bar{u}_{k-1} \bar{u}_{k-1}^{\prime}-\bar{u}_{k-2} \bar{u}_{k-2}^{\prime}\right)(s, y)\right| \frac{M \exp \left(-\frac{|x-y|^{2}}{M(t-s)}\right)}{\sqrt{2 \pi}(t-s)^{1 / 2}} d y d s .
\end{aligned}
$$

The conclusion follows by Gronwall's lemma.

\section{$\S 4.4$. Convergence of the sequence $\left\{u_{k}\right\}$}

We have that

$$
\begin{aligned}
& \int_{0}^{t} \bar{u}_{k}^{2}\left(s, X^{(t, x)}(s)\right) d_{\phi} W_{s} \\
& \quad=\int_{0}^{t} \bar{u}_{k}^{2}\left(s, X^{(t, x)}(s)\right) d^{-} W_{s}-\int_{0}^{t} \sigma \bar{u}_{k} \bar{u}_{k}^{\prime}\left(s, X^{(t, x)}(s)\right) d s .
\end{aligned}
$$

Therefore Propositions 9 and 10 applied to $u_{k+1}$ in (6) imply that as $k$ tends to $\infty$, the sequence $\int_{0}^{t} \bar{u}_{k}^{2}\left(s, X^{(t, x)}(s)\right) d_{\phi} W_{s}$ converges (in the $L^{2}(\Omega) \times L_{l o c}^{2}(\mathbb{R})$ sense) to the limit $\int_{0}^{t} \bar{u}^{2}\left(s, X^{(t, x)}(s)\right) d_{\phi} W_{s}$, uniformly in $t \in\left[0, t_{0}\right]$. Consequently we have $\lim _{k \rightarrow \infty} u_{k}(t, x)=\exists v(t, x)$ (uniformly in $t$ ), where $v$ is given by the

$$
v(t, x)=u_{0}\left(X^{(t, x)}(0)\right)+\nu \int_{0}^{t} \bar{u}^{2}\left(s, X^{(t, x)}(s)\right) d_{\phi} W_{s} .
$$


Since $E v(t, x)=\bar{u}(t, x)$ by virtue of the Proposition 11, we see that the limit $v(t, x)$ is the unique solution of the integral equation (4).

In fact suppose that we are given two solutions to (4), say $v_{1}$ and $v_{2}$, then by Proposition 11 one has that $E\left(v_{1}(t, x)\right)=E\left(v_{2}(t, x)\right)=\bar{u}(t, x)$. Therefore if one replaces this into the equation (4) one has that $v_{1}(t, x)=v_{2}(t, x)=u(t, x)$.

Thus we have shown the existence and uniqueness of the local solution $u(t, x), 0 \leq t \leq t_{0} \leq T$. The proof of the Theorem 4 is completed when we show that the discussion can be extended to the case $t \in\left[t_{0}, T\right]$. But this is an immediate consequence of the flow property below;

$$
u(t, x)=u\left(t_{0}, X^{(t, x)}\left(t_{0}\right)\right)+\nu \int_{t_{0}}^{t} \bar{u}^{2}\left(s, X^{(t, x)}(s)\right) d_{\phi} W_{s}, t \geq t_{0} .
$$

In order to carry this step one has to note that $u\left(t_{0}, x\right)$ is independent of $\sigma\left\{W_{s}-W_{t_{0}}, s \geq t_{0}\right\}$ and that $t_{0}<1$ is independent of all constants as stated in Lemma 6. Also the constants found in Lemmas 7 and 8 do not explode in a finite number of iterations therefore the extension of the previous arguments follow.

\section{§4.5. Verification of equation (5)}

It is trivial that the initial condition is satisfied as $\bar{u}(0, x)=E u_{0}\left(X_{0}^{(0, x)}\right)=$ $u_{0}(x)$. Repeating the same arguments as we have done previously, one proves that $\bar{u}(t, x)$ is two times differentiable in space. That is, one proves that the sequence $\bar{u}_{k}^{\prime \prime}$ converges uniformly and that its limit is $\bar{u}^{\prime \prime}(t, x)$. From (3) and (13) we have that $\bar{u}(t, x)$ is differentiable in time and

$$
\begin{aligned}
\frac{\partial \bar{u}}{\partial t}(t, x)= & \frac{1}{2} \sigma(t, x)^{2} \int_{\mathbb{R}} u_{0}(y) \frac{\partial^{2} p_{t, x}}{\partial x^{2}}(0, y) d y+b(t, x) \int_{\mathbb{R}} u_{0}(y) \frac{\partial p_{t, x}}{\partial x}(0, y) d y \\
& -\frac{\nu \sigma(t, x)^{2}}{2} \int_{0}^{t} d s \int_{\mathbb{R}} \sigma \bar{u} \bar{u}^{\prime}(s, y) \frac{\partial^{2} p_{t, x}}{\partial x^{2}}(s, y) d y \\
& -\nu b(t, x) \int_{0}^{t} d s \int_{\mathbb{R}} \sigma \bar{u} \bar{u}^{\prime}(s, y) \frac{\partial p_{t, x}}{\partial x}(s, y) d y \\
& -\nu \sigma(t, x) \bar{u} \bar{u}^{\prime}(t, x) \\
= & \frac{\sigma(t, x)^{2}}{2} \frac{\partial^{2} \bar{u}}{\partial x^{2}}(t, x)+b(t, x) \frac{\partial \bar{u}}{\partial x}(t, x)-\nu \sigma(t, x) \frac{\partial \bar{u}^{2}}{\partial x}(t, x) .
\end{aligned}
$$




\section{$\S 5$. Concluding Remarks}

Here we have obtained a new stochastic representation formula for the solution of the Burgers equation. This is called a Brownian particle equation. Its possible approximation process will require the use of a particle method to approximate the expectation in the equation. We hope that this representation will allow numerical simulation of the process $u$ and the study of other properties related with this process for small values of $\nu$. We have assumed here that the coefficients $a$ and $b$ are bounded but one could derive the above results with linear growth conditions doing appropriate changes in the arguments given for the proofs. In comparison with the stochastic representation obtained in [1] and [2] we do not require any integrability properties of $u_{0}$. In particular, one may have that $\int_{\mathbb{R}}\left|u_{0}(x)\right| d x=\infty$ and still the stochastic representation in Theorem 4 is valid.

\section{§6. Acknowledgements}

The authors express their thanks to the referee for a careful reading of the article. The second author was partially supported by grants BFM 2000807 and BFM 2000-0598. This research was done while the second author was visiting the Laboratory of Applied Mathematics and Stochastics at Kanazawa University.

\section{§7. Appendix}

Here we prove the following assertion

$$
\lim _{k \rightarrow \infty} \sqrt{k+1} \cdot B\left(\frac{k+1}{2}, \frac{1}{2}\right)=\sqrt{2 \pi}
$$

First, using the definition of the beta function we have that

$$
B\left(\frac{k+1}{2}, \frac{1}{2}\right)=\frac{\Gamma\left(\frac{1}{2}\right) \Gamma\left(\frac{k+1}{2}\right)}{\Gamma\left(\frac{k}{2}+1\right)}
$$

One studies the above quantities in the cases $k$ even and odd. We will do one case leaving the other for the reader. For $k=2 n, n \in N$, we have by properties of the gamma function that $\Gamma\left(\frac{k}{2}+1\right)=\frac{k}{2}$ ! and that

$$
\begin{aligned}
\Gamma\left(\frac{k+1}{2}\right) & =\frac{k-1}{2} \Gamma\left(\frac{k-1}{2}\right) \\
& =\frac{(k-1) ! !}{2^{k / 2}} \Gamma\left(\frac{1}{2}\right) \\
& =\frac{k !}{2^{k}\left(\frac{k}{2}\right) !} \Gamma\left(\frac{1}{2}\right)
\end{aligned}
$$


Therefore we have that

$$
B\left(\frac{k+1}{2}, \frac{1}{2}\right)=\frac{\Gamma\left(\frac{1}{2}\right)^{2} \frac{k !}{2^{k}\left(\frac{k}{2}\right) !}}{\left(\frac{k}{2}\right) !}
$$

Using Stirling's approximation for the factorial we have

$$
\begin{aligned}
B\left(\frac{k+1}{2}, \frac{1}{2}\right) & \sim \frac{\pi k^{k+1 / 2}}{\sqrt{2 \pi} 2^{k}\left(\frac{k}{2}\right)^{k+1}} \\
& \sim \frac{\sqrt{2 \pi}}{\sqrt{k}}
\end{aligned}
$$

In the other case $k=2 n+1$ one proceeds similarly finding the same limit.

The Gronwall inequality we used here is of some particular type due to the fact that the kernel function is degenerate. For this reason we give a brief account of this inequality.

Lemma 12. Let $y(t)$ be a real, non-negative function such that it satisfies

$$
y(t) \leq A_{1}+A_{2} \int_{0}^{t} \frac{y(s)}{\sqrt{t-s}} d s, \quad t \in[0, T],
$$

for two positive constants $A_{1}$ and $A_{2}$. Then

$$
y(t) \leq A_{1} \exp \left(2 A_{2} \sqrt{t}\right)
$$

The idea of the proof is to apply 6 . Theorem 1 in Mitrinović et al., page 358 for the kernel

$$
k(t, s)=\frac{1}{\sqrt{t-s+\epsilon}}
$$

obtain the Gronwall inequality that will depend on $\epsilon$ and then take limits as $\epsilon$ goes to 0 .

\section{References}

[1] Bossy, M. and Talay, D., A stochastic particle method for the McKean-Vlasov and the Burgers equation. Math. Comp., 66-217 (1997), 157-192.

[2] Convergence rate for the approximation of the limit law of weakly interacting particles: application to the Burgers equation. Ann. Appl. Probab., 6 (1996), 818-861.

[3] Friedman, A., Partial differential equations of parabolic type, Prentice-Hall, 1964. 
[4] Ikeda, N., and Watanabe, S., Stochastic Differential Equations and Diffusion Processes, Amsterdam Oxford New York, North-Holland/Kodansha, 1989.

[5] Mitrinovic, D. S., Pecaric, J. E. and Fink, A. M., Inequalities Involving Functions and Their Integrals and Derivatives, Kluwer Academic Publishers, 1991.

[6] Ogawa, S., On a class of SPDEs called the Brownian particle equation - Model for nonlinear diffusions, Monte Carlo Methods Appl., 7 (2001), 321-328.

[7] , On the Brownian particle equations and the noncausal stochastic calculus, Rend. Acad. Naz. Sci. XL, 119 XXV, (2001), 125-139.

[8] ${ }_{-}$, On the numerical simulation of nonlinear SDEs and Burgers like processes, Proceedings of The Workshop on Turbulent Diffusion and Related Problems in Stochastic Numerics (at ISM in Oct 1996) (ISM Joint Research Report 99), pp. 140-159, 1997.

[9] , Topics in the theory of noncausal stochastic calculus, in Diffusion Processes and Related Problems in Analysis, 1, ed. Mark Pinsky, Birkhäuser Boston Inc., pp. 411-420, 1990.

[10] integrals, Japan J. Appl. Math., 2 (1985), 229-240.

[11] $\ldots$, Sur le produit direct du bruit blanc par lui-même, C. R. Acad. Sci. Sér. A, 288 (1979), 359-362.

[12] , A partial differential equation with the white noise as a coefficient, $Z$. W. verw. Geb., 28 (1973), 53-71.

[13] , On a Riemann definition of the stochastic integral, I, II, Proc. Japan Acad., 46 (1970), 153-156, ibid. 157-161. 